
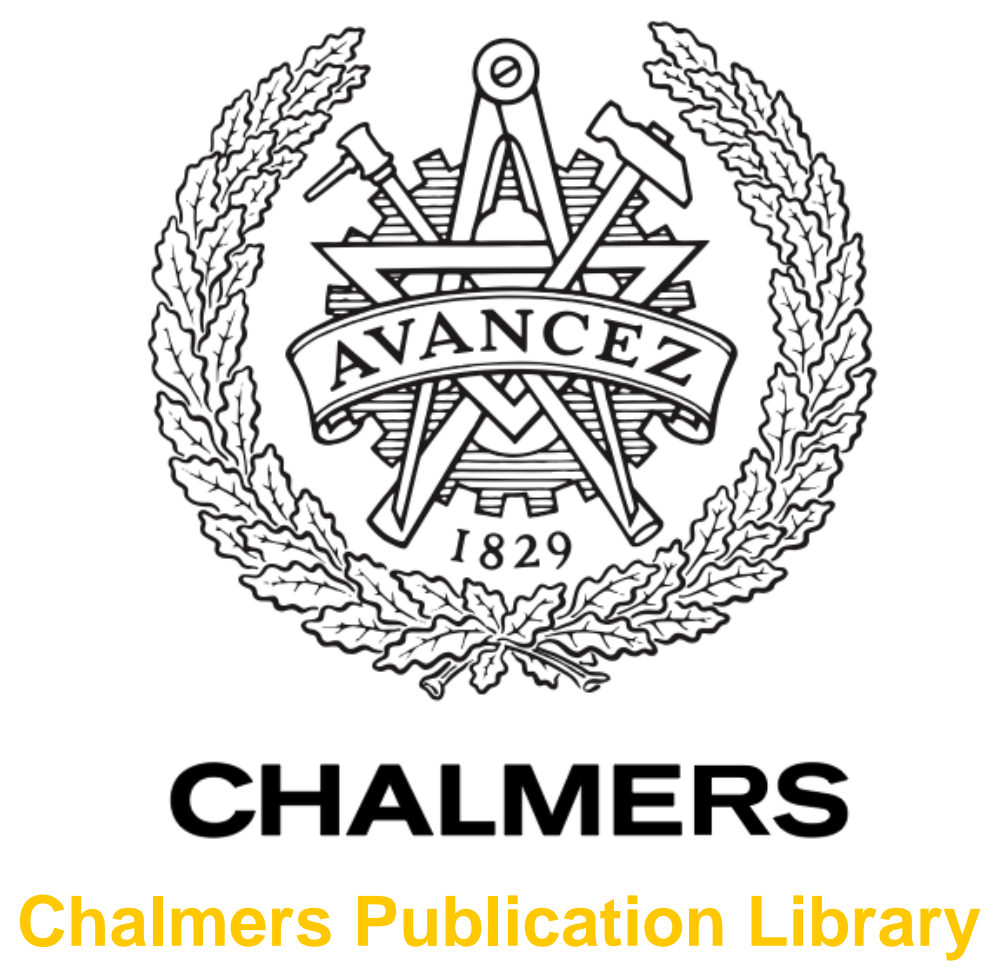

\title{
A Real-time MHE and NMPC Scheme for Wind Turbine Control
}

This document has been downloaded from Chalmers Publication Library (CPL). It is the author's version of a work that was accepted for publication in:

\section{Conference on Decision and Control 2013}

Citation for the published paper:

Gros, S. ; Vukov, M. ; Diehl, M. (2013) "A Real-time MHE and NMPC Scheme for Wind Turbine Control". Conference on Decision and Control 2013 pp. 1017 - 1022.

Downloaded from: http://publications.lib.chalmers.se/publication/194403

Notice: Changes introduced as a result of publishing processes such as copy-editing and formatting may not be reflected in this document. For a definitive version of this work, please refer to the published source. Please note that access to the published version might require a subscription. 


\title{
A Real-time MHE and NMPC Scheme for Wind Turbine Control
}

\author{
Sébastien Gros, Milan Vukov, Moritz Diehl
}

\begin{abstract}
Model Predictive Control (MPC) is a strong candidate for the control of large Multi-Mega Watt Wind Turbine Generators (WTG). Several MPC and some Nonlinear MPC schemes have been proposed in the literature, formulating the problem of balancing the power generation against the structural and actuator fatigue through a reference-tracking scheme. While the resulting schemes offer very promising results in term of load reduction, especially when reliable LIDAR systems are available, no NMPC scheme fast enough to achieve a real-time implementation has yet been proposed. This paper presents such a real-time NMPC scheme. Moreover, the proposed scheme maximizes directly the power generation, as opposed to tracking the optimal wind-dependent WTG steady-state.
\end{abstract}

Keywords : real-time NMPC and MHE, wind turbine control.

\section{INTRODUCTION}

With the size of wind turbine generators (WTG) steadily increasing, there has been a growing interest in alleviating structural fatigue through better control [4]. For multimegawatt WTG (MMWTG) in particular, fatigue load on the tower has received special attention since the cost of the foundations can contribute a significant part of a WTG total cost, up to $40 \%$ for an off-shore wind turbine [24]. The control of structural fatigue has mainly focused on the tower fore-aft oscillations that have a direct impact on the WTG foundations. Such oscillations are commonly reduced through collective pitch control, using linear feedback based on the measurements of the WTG nacelle fore-aft acceleration [3].

Model Predictive Control (MPC) techniques are emerging as strong candidates for the control of future Multi-Megawatt Wind Turbine Generators (MM-WTG). Among the main motivations to adopt advanced control techniques for WTG control are: the tighter actuator limitations, the intricate operational strategies, the introduction of more actuators, the dynamic nonlinearities, the developing regulations on grid compatibility, and the predictive information gathered through LIDAR systems [21]. Promising results have been obtained in simulations, showing the potential of MPC and Nonlinear MPC techniques when compared to more classical control approaches [22], [15], [23]. However, a NMPC scheme that is fast enough to achieve a real-time implementation has not yet been proposed in the literature. Presenting such a scheme is the main purpose of this paper.

NMPC schemes for wind turbine control are commonly use tracking objectives as a surrogate for maximizing the

S. Gros, M. Vukov and M. Diehl are with the Optimization in Engineering Center (OPTEC), K.U. Leuven, Kasteelpark Arenberg 10, B-3001 LeuvenHeverlee, Belgium. sgros@esat.kuleuven.be power capture. This approach offers good performances, albeit it has some drawbacks, chiefly in term of controller tuning. In contrast, this paper presents an NMPC scheme which maximizes directly the power capture (see the companion paper [12] for more details).

Because it impinge directly on the control performances, the estimation of the WTG state is an important issue in the context of NMPC. Previous works have either considered that a full-state measurement is available, or have relied on an Extended Kalman Filter (EKF). In this paper, a MovingHorizon Estimation (MHE) scheme [20] is used, where the nonlinear dynamics of the system are explicitly taken into account.

The contribution of this paper is twofold: 1) it shows that MHE/NMPC can achieve the computational speed required for a deployment on real WTGs, and 2) it presents a realtime implementation of an NMPC formulation that directly maximizes the power capture, and allows for efficiently treating the WTG operational constraints while avoiding recursive feasibility issues. The details of the proposed formulation are developed in the companion paper [12].

This paper is organized as follows. Section II briefly presents the WTG model used in this paper. Section III presents the real-time NMPC scheme. Section IV presents the real-time MHE scheme. Section V provides the details of the real-time implementation. Section VI presents simulation results. Conclusion and future developments are proposed in Section VII.

\section{Wind TURBINE MODEL}

The model presented in [22] is also used in this paper. It is briefly recalled here:

$$
\begin{aligned}
J \dot{\omega} & =T_{\text {aero }}-r_{\mathrm{g}}^{-1} T_{\mathrm{g}}, \\
\ddot{x} & =M^{-1}\left(F_{\text {aero }}-C \dot{x}-K x\right), \\
T_{\text {aero }} & =\frac{1}{2} \rho A C_{P}(\beta, \lambda) \frac{V^{3}}{\omega}, \\
F_{\text {aero }} & =\frac{1}{2} \rho A C_{T}(\beta, \lambda) V^{2}, \\
\lambda & =\frac{R \omega}{V}, \quad V=W-\dot{x},
\end{aligned}
$$

where $\omega$ is the rotor speed, $x$ the nacelle fore-aft position, $\beta$ the collective pitch angle of the blades, $T_{\text {aero }}$ the aerodynamic torque perceived by the rotor, $F_{\text {aero }}$ is the aerodynamic force perceived by the hub, $W$ is the time-dependent wind speed and $V$ is the relative wind speed at the hub. Parameters $\rho$ and $A$ are the air density and the rotor area respectively. Factor $\lambda$ is the tip-speed ratio, which plays an important role in the aerodynamics of WTG. 
TABLE I

MODEL PARAMETERS

\begin{tabular}{|c||c|l|}
\hline Parameter & Description & Value \\
\hline$J$ & Total rotor inertia & $40.47 \cdot 10^{6}\left[\mathrm{~kg} / \mathrm{m}^{2}\right]$ \\
$r_{\mathrm{g}}$ & Gearbox ratio & $1 / 97[-]$ \\
$M$ & Tower fore-aft inertia & $0.43 \cdot 10^{6}[\mathrm{~kg}]$ \\
$C$ & Tower damping & $1.76 \cdot 10^{4}[\mathrm{Ns} / \mathrm{m}]$ \\
$K$ & Tower stiffness & $1.77 \cdot 10^{6}[\mathrm{~N} / \mathrm{m}]$ \\
$\rho$ & Air density & $1.23\left[\mathrm{~kg} / \mathrm{m}^{3}\right]$ \\
$A$ & Rotor area & $1.25 \cdot 10^{4}\left[\mathrm{~m}^{2}\right]$ \\
$R$ & Rotor radius & $63[\mathrm{~m}]$ \\
\hline
\end{tabular}

Parameter $J=J_{\mathrm{r}}+r_{\mathrm{g}}^{-2} J_{\mathrm{g}}$ is the total turbine inertia as seen from the rotor, where $J_{\mathrm{r}}$ is the rotor inertia, including the hub and the blades, $J_{\mathrm{g}}$ is the generator inertia, and $r_{\mathrm{g}}$ is the gearbox ratio. The state and the control input vectors $X \in \mathbb{R}^{2}$ and $U \in \mathbb{R}^{2}$ are given by:

$$
X=\left[\begin{array}{llllll}
\omega & x & \dot{x} & T_{\mathrm{g}} & \beta & \dot{\beta}
\end{array}\right], \quad U=\left[\begin{array}{ll}
\dot{T}_{\mathrm{g}} & \ddot{\beta}
\end{array}\right] .
$$

In the following, the dynamics (1) are lumped into the function $f$ :

$$
\dot{X}=f(X, U, W) .
$$

Ignoring the tower fore-aft motion, the aerodynamic power harvested from the wind field reads:

$$
P_{\text {aero }}=\frac{1}{2} \rho A C_{P}(\beta, \lambda) W^{3},
$$

while the generated power reads:

$$
P=\eta r_{\mathrm{g}}^{-1} T_{\mathrm{g}} \omega
$$

where $\eta<1$ is the generator efficiency. The model parameters are summarized in table I.

The maximum aerodynamic efficiency is achieved by maximizing $C_{P}$. At steady-state, it is yielded by the windindependent optimal values $\beta^{*}$ and $\lambda_{\text {opt }}$ achieving the maximal value of $C_{P}$, labeled $C_{P}^{\max }$. The optimal steady-state rotor speed $\omega_{\mathrm{opt}}$ and rotor torque $T_{\text {aero }}^{\mathrm{opt}}$ then satisfy:

$$
W=\frac{R \omega_{\mathrm{opt}}}{\lambda_{\mathrm{opt}}}, \quad T_{\mathrm{aero}}^{\mathrm{opt}}=\frac{1}{2} \rho A C_{P}^{\max } \frac{W^{3}}{\omega_{\mathrm{opt}}} .
$$

It follows that:

$$
T_{\mathrm{g}}^{\mathrm{opt}}=r_{\mathrm{g}} T_{\mathrm{aero}}^{\mathrm{opt}}=K \omega_{\mathrm{opt}}^{2}
$$

with $K=\frac{1}{2} r_{\mathrm{g}} \rho A C_{P}^{\max }\left(\frac{R}{\lambda_{\text {opt }}}\right)^{3}$. In fact, classical WTG controllers commonly apply the steady-state optimal relationship (3) as a stabilizing feedback law (see e.g. [18]), i.e. they apply $T_{\mathrm{g}}=K \omega^{2}$ in order to achieve a state trajectory reasonably close to its dynamic optimum.

\section{NMPC SCHEME}

In this paper, the objective function of the NMPC scheme seeks to achieve the best trade-off between the power capture, the structural load, and the pitch activity, while respecting the WTG operational constraints. It is presented next.

\section{A. Power capture \& load alleviation}

NMPC for wind turbine control is a multi-objective optimization problem, which seeks at achieving an economically sound trade-off between maximizing the power capture, and minimizing the structural fatigue and the pitch activity. In this paper, the objective function associated to the power optimization was chosen as the aerodynamic power (2), using the formulation developed in [12], which presents a polynomial interpolation of the $C_{P}$ coefficient suitable for a Gauss-Newton Hessian approximation.

A classical quadratic penalty on the nacelle fore-aft velocity $\dot{x}_{\mathrm{T}}$ was chosen for the reduction of the tower load. Whether this choice best reflects the actual structural fatigue or whether a more accurate penalty could be devised is the object of current research. In the NMPC objective function, the terms associated to the power maximization and load alleviation read:

$$
\Phi_{0}=\int_{t}^{t+T_{\mathrm{c}}}\left(Q_{\text {Load }} \dot{x}^{2}-P_{\text {aero }}\right) d \tau .
$$

\section{B. Operational strategy}

The rotor speed and generator torque of WTG are subject to soft operational constraints, i.e. constraints that ought not be excessively violated, which are collectively referred to as the operational strategy (see e.g. [17], [6], and Fig. 1). The operational constraints considered here are further detailed in [12].

Additionally to the operational constraints, limitations on the pitch angle, rate and acceleration must be respected. Moreover, the limited capabilities of the power electronics prohibit very fast variations of the generator torque.

Classical WTG control techniques typically rely on elaborate switching strategies and filter techniques to track the operational strategy (see e.g. [16]), so as to maximize the power capture while respecting the constraints. Classical NMPC schemes use switching references and weights [22]. In this work, the operational strategy and actuator limitations are handled in the form constraints, summarized here:

$$
\begin{aligned}
& \omega^{\text {cut }- \text { in }} \leq \omega \leq \omega^{\text {rated }} \\
& P \leq P_{\text {rated }} \\
& 0 \leq T_{\mathrm{g}} \leq T_{\mathrm{g}}^{\text {max }} \\
&-45 \mathrm{kNm} \cdot \mathrm{s}^{-1} \leq \quad \dot{T}_{\mathrm{g}} \leq 45 \mathrm{kNm} \cdot \mathrm{s}^{-1} \\
& \beta^{*} \leq \beta \leq 30 \mathrm{deg} \\
&-7 \mathrm{deg} \cdot \mathrm{s}^{-1} \leq \dot{\beta} \leq 7 \mathrm{deg} \cdot \mathrm{s}^{-1} \\
&-20 \mathrm{deg} \cdot \mathrm{s}^{-1} \leq \ddot{\beta} \leq 20 \mathrm{deg} \cdot \mathrm{s}^{-1}
\end{aligned}
$$

\section{Constraint relaxation}

Because of the system perturbations and state estimation error, the recursive feasibility of some of the state constraints is not guaranteed. Because the evolution of $\omega$ is subject to wind perturbations and model errors, constraints (4)-(5) are 


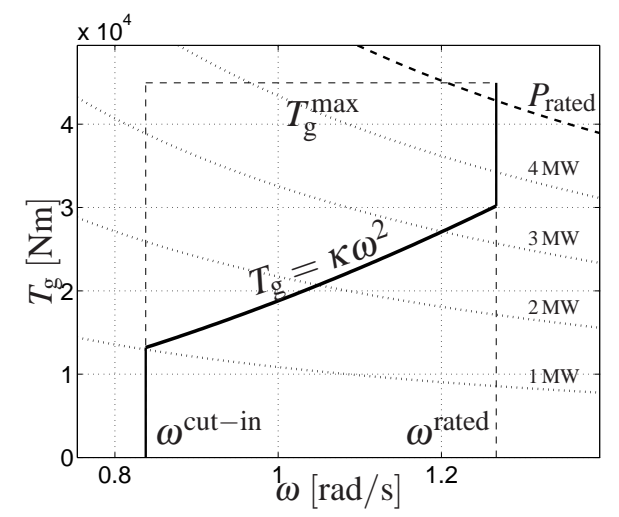

Fig. 1. Conventional operational strategy. The generator torque and the rotor speed are subject to box constraints. The thick non-smooth curve displays the steady-state optimal relationship between the rotor speed and generator torque. The light dotted lines are curves of constant power.

particularly problematic. This problem can be corrected by using a slack reformulation of the problematic constraints:

$$
\begin{aligned}
\omega^{\text {cut-in }}-S_{\omega} \leq \omega \leq \omega^{\text {rated }}+S_{\omega}, & S_{\omega} \geq 0 \\
P \leq P_{\text {rated }}+S_{P} & S_{P} \geq 0
\end{aligned}
$$

where $S_{\omega}, S_{P} \in \mathbb{R}^{+}$, labelled slack variables (see e.g. [5], p. 131), are time-dependent variables assimilated as control inputs in the NMPC scheme, which are penalized in the NMPC objective function, such that any violation of the constraint results in an extra cost. In this paper, the penalty on the slack variables was chosen as the quadratic form:

$$
\Pi_{S}=Q_{\omega}\left(S_{\omega}+S_{\omega}^{0}\right)^{2}+Q_{P}\left(S_{P}+S_{P}^{0}\right)^{2}
$$

with $S^{0} \in \mathbb{R}^{+}$, hence imposing a mixed $L_{1} / L_{2}$ penalty on constraints violations. See [12] for more details and a discussion on the selection of the weights in (12).

\section{Limitation of the cumulated constraint violation}

While relaxing state constraints is often crucial to ensure the recursive feasibility of the NMPC scheme, it is often also crucial to ensure that the violation does not last over time. E.g. the constraint on the maximum generated power is typically associated to the limited capabilities of the power electronics and generator at dissipating heat. It follows that an over-rated power can be tolerated provided that it does not overrun the heat dissipation capabilities of the generator and power electronics. Such a requirement can be formulated as:

$$
\sigma_{P} \leq \sigma_{P}^{\max }, \quad \dot{\sigma}_{P}=S_{P}-\alpha \sigma_{P}
$$

where $\sigma_{P}$ is a differential state accounting for the accumulated constraint violation over time, and coefficient $\alpha$ is a dissipative term accounting for how fast the system recovers from an accumulated constraint violation. In fact, in the case of generated power, it can be verified that $\sigma_{P}$ is the excess of thermal energy accumulated in the electromechanical components of the WTG, and $\alpha$ is the coefficient of thermic dissipation in $\mathrm{s}^{-1}$. Parameter $\sigma_{P}^{\max }$ ought to be chosen as

$$
\sigma_{P}^{\max }=\frac{P_{\max }-P_{\text {rated }}}{\alpha}
$$

where $P_{\max }$ is the maximal steady-state power that can be tolerated by the electro-mechanical components of the WTG, in Joules. Additionally, in order to prevent unnecessary activations of the constraint (13), a $L_{2}$ penalty:

$$
\Pi_{\sigma}=Q_{\sigma_{P}} \sigma_{P}^{2}
$$

is introduced in the cost function, with $Q_{\sigma_{P}}$ proportional to $W^{3}$. As a result, any accumulation of over-rated power is penalized.

\section{E. Regularization}

The regularization of the variations of the generator torque is performed via penalizing the variations of generated power (see [12] for more details). The proposed regularization of $\dot{T}_{\mathrm{g}}$ reads:

$$
R_{\dot{P}}=Q_{\dot{P}}\left\|\dot{P}_{\text {elec }}\right\|^{2}=Q_{\dot{P}}\left\|\eta r_{\mathrm{g}}^{-1}\left(\dot{T}_{\mathrm{g}} \omega+T_{\mathrm{g}} \dot{\omega}\right)\right\|^{2} .
$$

A penalty on the power variations tends to push the WTG toward following the curves of constant power (see Fig. 1).

The regularization of the pitch rate $\dot{\beta}$ and pitch acceleration $\ddot{\beta}$ need to reflect the actuator fatigue generated by the pitch activity. A common and reasonable choice of regularization for the pitch activity reads:

$$
R_{\beta}=Q_{\dot{\beta}} \dot{\beta}^{2}+Q_{\ddot{\beta}} \ddot{\beta}^{2}
$$

In this paper, the weights in (16) were chosen constant. A systematic choice of these weights and their systematically balancing them against the structural penalty $Q_{\mathrm{Load}}$ is the object of future research.

\section{F. NMPC formulation}

The NMPC scheme was formulated as follows:

$$
\begin{aligned}
\min _{U, X, S_{\omega}, S_{P}} & \Phi_{0}+\int_{t}^{t+T_{\mathrm{c}}}\left(\Pi_{S}+\Pi_{\sigma}+R_{\dot{P}}+R_{\beta}\right) d \tau \\
\text { s.t. } & \dot{X}=f(X, U, W), \quad \dot{\sigma}_{P}=S_{P}-\alpha \sigma_{P} \\
& (11) \quad h(X, U) \leq 0
\end{aligned}
$$

where $h(X, U) \leq 0$ lumps together the hard constraints (6)(10), and the predicted, time-dependent wind speed profile $W$ is provided by the LIDAR system.

\section{MHE SCHEME}

In this paper, it is proposed to perform the state estimation based on Moving Horizon Estimation. The scheme used in this paper can be summarized as follows:

$$
\begin{aligned}
\min _{X, U, W} & \frac{1}{2} \int_{t-T_{\mathrm{e}}}^{t}\left(\|Y(X, W)-\bar{Y}(\tau)\|_{Q_{\mathrm{E}}}^{2}+\left\|U-U_{\mathrm{MPC}}\right\|_{R_{\mathrm{E}}}^{2}\right) d \tau \\
\text { s.t. } & \dot{X}=f(X, U, W)
\end{aligned}
$$

where the vector of measurements includes the rotor velocity, nacelle acceleration, pitch angle and past LIDAR wind measurements:

$$
\bar{Y}=\left[\begin{array}{lllll}
\omega & \ddot{x} & \beta & \dot{\beta} & W
\end{array}\right],
$$


TABLE II

AVAILABLE SENSORS FOR MHE

\begin{tabular}{|c||c|l|}
\hline Sensor & Measurement & $\sigma$ \\
\hline Rotor speed & $\omega$ & $1 \mathrm{rad} \cdot \mathrm{s}^{-1}$ \\
Tower acc. & $\ddot{x}$ & $10^{-2} \mathrm{~cm} \cdot \mathrm{s}^{-2}$ \\
Pitch angle & $\beta$ & $1 \mathrm{deg}$ \\
Pitch rate & $\beta$ & $1 \mathrm{deg} \cdot \mathrm{s}^{-1}$ \\
LIDAR & $W$ & $0.5 \mathrm{~m} \cdot \mathrm{s}^{-1}$ \\
\hline
\end{tabular}

matrices $Q_{\mathrm{E}}, Q_{\mathrm{W}}$ are diagonal matrices, with the inverse of the measurement covariances (see Tab. II) as diagonal entries, and matrix $R_{\mathrm{E}}$ accounts for the input covariances, and:

$$
R_{\mathrm{E}}=\operatorname{diag}\left(\left[\begin{array}{ll}
7 \cdot 10^{-2} & 10^{-4}
\end{array}\right]\right) .
$$

in $[\mathrm{deg}]$ and $[\mathrm{kNm}]$ respectively.

\section{REAL-TIME IMPLEMENTATION}

This section presents the algorithmic components used to deploy in real time the NMPC and MHE schemes presented in Sections III-IV.

\section{A. Multiple-shooting}

Because the model dynamics $\dot{X}=f(X, U)$ are nonlinear and because a prediction horizon $T_{\mathrm{c}}$ of several seconds needs to be considered, the optimization shall be performed using simultaneous approaches [1]. In this paper, a direct multiple-shooting [2] approach was chosen, where the model dynamics are discretized on a uniform time grid $t_{0}, \ldots, t_{N}$ via numerical integration over the time intervals $\left[t_{k}, t_{k+1}\right]$. The control input $U$ is discretized as piecewise constant $U_{0}, \ldots, U_{N-1}$ over the time intervals. The inequality constraints are discretized on the same time grid.

After each iteration, the initial guesses for problems (17), (18) are updated by discarding the first element, shifting the state and input vectors $X, U$, and adding a new element at the end of the prediction horizon. The control inputs are updated by duplicating the last element, and the states are updated using a forward integration on the last time interval [8].

\section{B. Parametric embedding \& Real-time iteration}

Both the initial value $X(t)$ and the wind profile $W([t, t+$ $\left.\left.T_{\mathrm{c}}\right]\right)$ predicted by the LIDAR are embedded in the NMPC scheme (17), and the latest measurements $Y(t)$ are embedded in the MHE scheme (18). As a result, the first Newton step provides already a good approximation of the exact solutions to both problem (17) and (18), hence a real-time iteration (RTI) approach is used, where only one full Newton step per control time instant is performed [7].

Moreover, most computations required to perform the first Newton step can be carried out before the new initial conditions $X(t)$ and measurements $Y$ are known. As a result, the re-calculation of the control inputs can be prepared before the new measurement and state estimation is known, and finished in a very short time once they become available, hence reducing significantly the delay between state estimation and control in the NMPC scheme [7].

\section{Implicit integration scheme}

It was obsvered in simulations that using an explicit integration scheme such as a Runge-Kuta 4 requires a large number of steps per control interval to achieve a high level of accuracy, while the implicit integration scheme presented in [19] provides a good level of accuracy with a very low number of steps per control interval, allowing for dramatically reducing the computational time required for the integration of the system dynamics (see Fig. 8). Additionally the implicit integration scheme presented in [19] allows for performing MHE using sensor data sampled at a very high frequency, and is therefore ideal to efficiently use the data provided by the accelerometer and the encoders.

\section{Code generation \& Software approach}

The compiled code is automatically tailored to the problem considered, and contains only the absolutely essential algorithmic components. Based on a symbolic representation of the optimal control problem to be solved online, problemspecific structures such as dimensions, and sparsity patterns are exploited during the code generation process to avoid irrelevant and redundant computations (see [14], [11]). Solutions to the NMPC and MHE schemes were computed based on the software ACADO Toolkit [13], which implements direct multiple-shooting and RTI. The underlying parametric Quadratic Programs (QPs) are condensed and solved using a dense online active set strategy implemented in the software qpOASES [10]. The generated code was executed on an i7 $2.66 \mathrm{GHz} / 64$ bits.

\section{Simulation RESUlts}

The simulations were performed using the NMPC and MHE schemes presented in Sections III- IV, and the algorithmic components presented in Section V. The control parameters are provided in Tab. III. The penalties associated to the constraint relaxation must be chosen sufficiently large to prevent undesired constraint violations, but are not critical for the performance of the controller, and therefore easy to tune. The wind profile is displayed in Fig. 2. The resulting state trajectories are displayed in Fig. 3. The resulting generated power $P$ and the aerodynamic power $P_{\text {aero }}$ are displayed in Fig. 4, both as a time series and as a power curve. It can be observed that the optimal power is achieved in the below-rated region (lower graph). The generated power can temporarily exceed the rated power (upper-graph), resulting in an activation of the slack variable $S_{P}$ (Fig. 5. lower graph), and an accumulation $\sigma_{P}$ (Fig. 5. upper graph). The resulting $\omega-T_{\mathrm{g}}$ trajectories are reported in the operational strategy graph (Fig. 6). It can be observed that the NMPC scheme, by tracking the optimal power capture and regularizing the variations of power generation, departs significantly from the steady-state optimum manifold $T_{\mathrm{g}}=\kappa \omega^{2}$. The achieved computational times for the NMPC and MHE schemes are reported in Fig. 7, and are consistently lower than the chosen sampling time $T_{\mathrm{S}}=0.2 \mathrm{~s}$. The simulation has been performed for different number of integrator step per shooting interval, using both an explicit Runge-Kutta 4 and the 
TABLE III

CONTROL PARAMETERS

\begin{tabular}{|c||c|l|}
\hline Parameter & Description & Value \\
\hline$T_{\mathrm{c}}=T_{\mathrm{e}}$ & Horizons (NMPC \& MHE) & $10 \mathrm{~s}$ \\
$T_{\mathrm{S}}$ & Sampling time (NMPC \& MHE) & $0.2 \mathrm{~s}$ \\
$Q_{\omega}, Q_{P}$ & Weights for $\Pi_{S}$, see (12) & $5 \cdot 10^{-1}, 5 \cdot 10^{-2}$ \\
$S_{\omega}^{0}, S_{P}^{0}$ & Ref. for $\Pi_{S}$, see (12) & 1,1 \\
$P_{\max }$ & Max. power (Sec. III-D) & $1.01 \cdot P_{\text {rated }}$ \\
$Q_{\sigma_{P}}, \sigma_{P}^{0}$ & Weights \& ref. for $\Pi_{\sigma_{P}}$, see (14) & $5 \cdot 10^{-2},-1$ \\
$Q_{\dot{P}}, Q_{\dot{\beta}}, Q_{\ddot{\beta}}$ & Weights for $\Pi_{\dot{P}} \& \Pi_{\beta}(15) \&(16)$ & $1,0,10^{-3}$ \\
$Q_{\text {Load }}$ & Weight for the tower motion $\dot{x}$ & $10^{3}$ \\
\hline
\end{tabular}

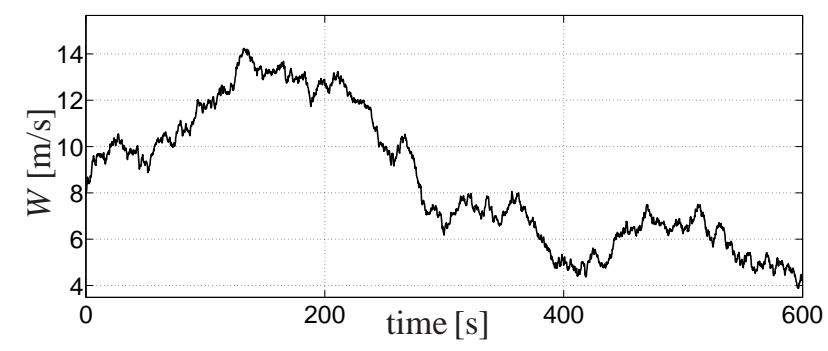

Fig. 2. $10 \mathrm{~min}$ wind profile

implicit integrator presented in [19]. The average integration accuracy vs. the number of steps and vs. the overall NMPC computational time are reported in Fig. 8, showing that the implicit integrator performs significantly better than the explicit scheme. The number of QP iterations and the KKT conditions over time are reported in Fig. 9.

\section{CONCLUSION \& FURTHER CONSIDERATION}

This paper has presented a real-time MPC/MHE scheme for the control of WTGs. The presented scheme maximizes directly the captured power as opposed to using a more conventional reference-tracking scheme as a proxy. The operational constraints (speed, power, torque and actuator limitations) of the WTG were treated in the form of relaxed
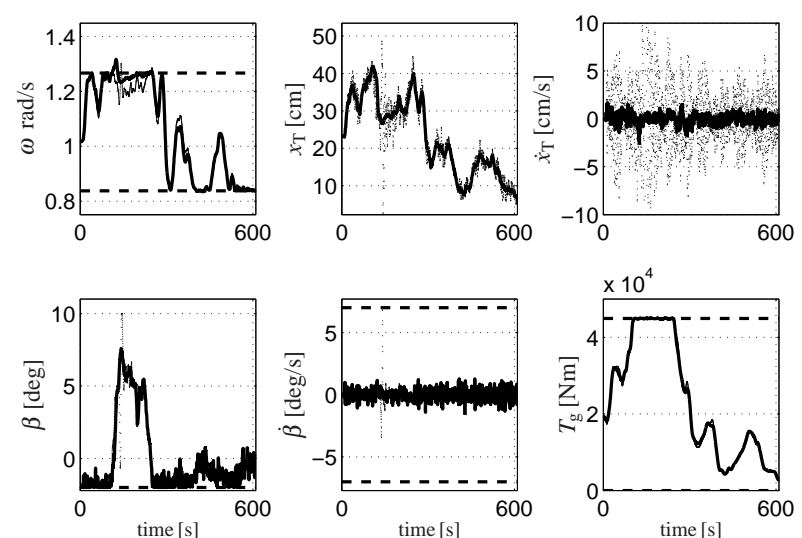

Fig. 3. Time series for the rotor speed, tower position and velocity, pitch angle and pitch rate, and generator torque, with bounds. The rotor speed $\omega$ (upper-left graph) is allowed to slightly violate its bounds. The light-grey curves report the same simulation with a tower weight $Q_{\text {Load }}=0$.
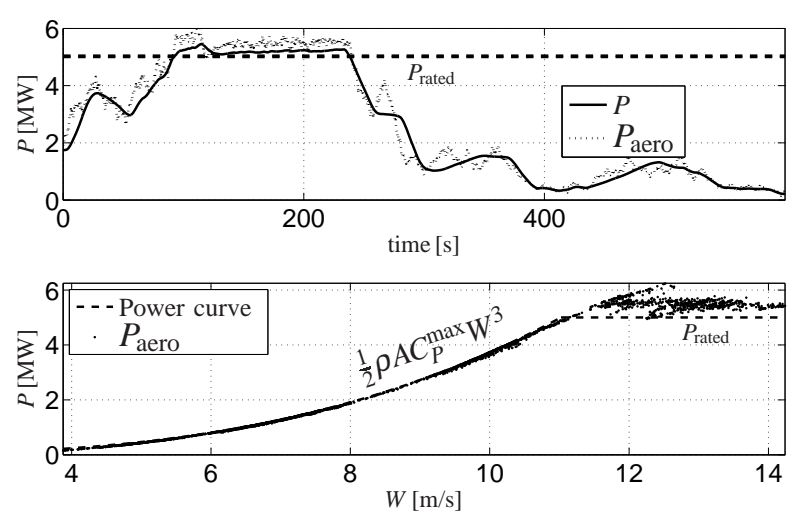

Fig. 4. The time series of the aerodynamic and generated power can be observed in the upper graph. The NMPC scheme tracks efficiently the optimal power curve $P=\frac{1}{2} \rho A C_{P}^{\max } W^{3}$ in the below-rated wind speed region (lower graph).
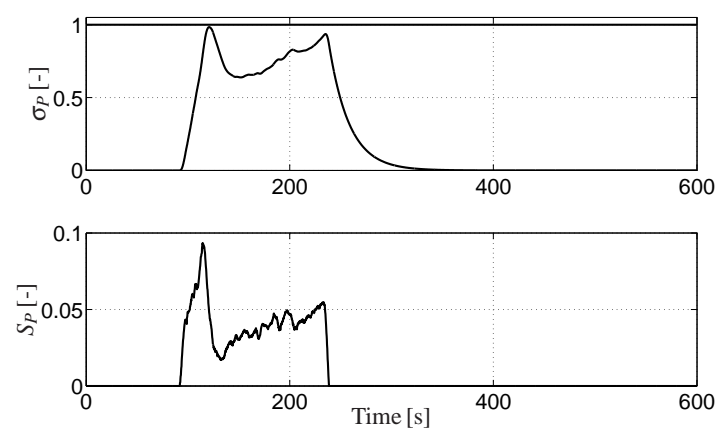

Fig. 5. The slack variable $S_{P}$ (lower graph) is activated whenever $P>P_{\text {rated }}$. The cumulated power excess is monitored via $\sigma_{P}$ (upper graph) and kept within its limit (scaled to 1)

constraints. A regularization of the generated power is proposed, so as to provide a means to include the power quality in the objective function.

It has been observed in this work that a remaining difficulty is to provide an accurate interpolation of the $C_{P}$ table over the whole operational range, using a single polynomial function of $\beta$ and $\lambda$. This difficulty is not intrinsic to the proposed scheme, and can be solved by a fast, codegenerated interpolation algorithm which outputs the function sensitivities and guarantees a sufficient smoothness. See [12] for a more detailed discussion. Such an algorithm is the object of future research.

Finally, for horizons longer than $10 \mathrm{~s}$, it may be advantageous to consider using a sparse solver QP solver, such as an interior-point solver, whose computational complexity increases linearly with the horizon length instead of cubically.

\section{ACKNOWLEDGMENTS}

This research was supported by Research Council KUL: PFV/10/002 Optimization in Engineering Center OPTEC, GOA/10/09 MaNet; Flemish Government: IOF/KP/SCORES4CHEM, FWO: PhD/postdoc grants and projects: G.0320.08 (convex MPC), G.0377.09 (Mechatronics MPC); IWT: PhD Grants, projects: SBO LeCoPro; Belgian Federal Science Policy Office: IUAP P7 (DYSCO, Dynamical systems, control and optimization, 2012-2017); EU: FP7-EMBOCON (ICT-248940), FP7-SADCO ( MC ITN-264735), ERC ST HIGHWIND (259 166), Eurostars SMART, ACCM. 


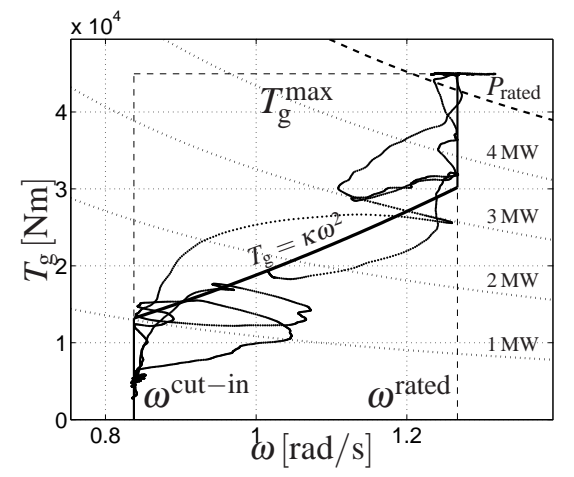

Fig. 6. Operational strategy. Maximizing the power capture and regularizing the variations of generated power $P$ yields $\omega-T_{\mathrm{g}}$ trajectories that diverge significantly from the steady-state optimum manifold $T_{\mathrm{g}}=\kappa \omega^{2}$.
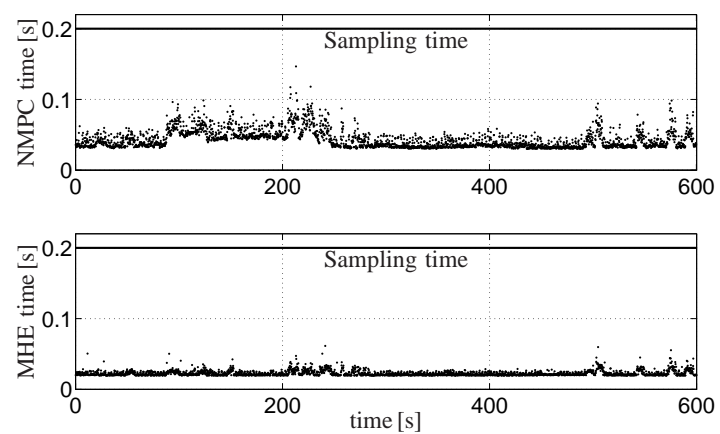

Fig. 7. Computational time of the NMPC and MHE schemes at each sampling instants. The computational time of both schemes is consistently and safely below the actual sampling time.
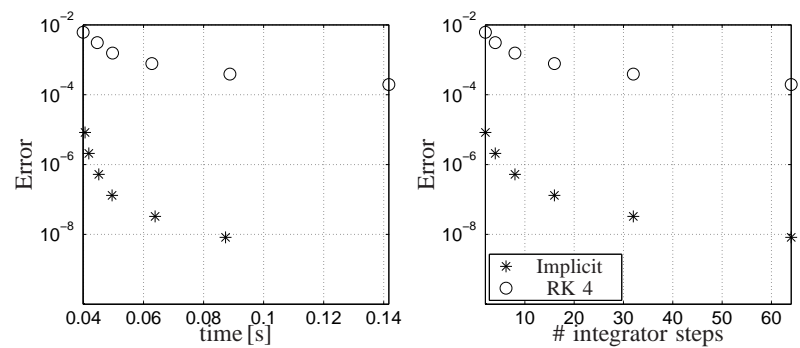

Fig. 8. Average integration error vs. average overall NMPC computational time (left graph), and vs. \# of steps per control interval (right graph) for the Runge-Kutta 4 and the implicit integrator.
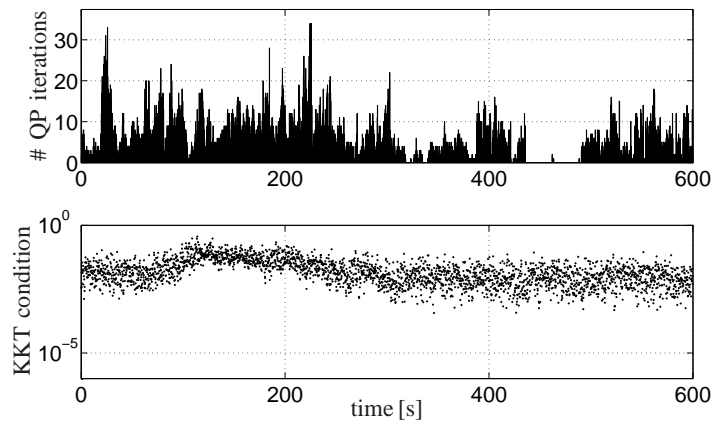

Fig. 9. Number of iterations of the primal active set QP solver (upper graph). The KKT conditions (lower graph) remain low despite an RTI approach is used.

\section{REFERENCES}

[1] L.T. Biegler. An overview of simultaneous strategies for dynamic optimization. Chemical Engineering and Processing, 46:1043-1053, 2007.

[2] H.G. Bock and K.J. Plitt. A multiple shooting algorithm for direct solution of optimal control problems. In Proceedings 9th IFAC World Congress Budapest, pages 243-247. Pergamon Press, 1984.

[3] E. A. Bossanyi. Wind turbine control for load reduction. Wind Energy, 6(3):229244, 2003.

[4] E. A. Bossanyi. Further Load Reductions with Individual Pitch Control. Wind Energy, 8:481-485, 2005.

[5] S. Boyd and L. Vandenberghe. Convex Optimization. University Press, Cambridge, 2004.

[6] Burton, T., Sharpe, D., Jenkins, N. and Bossanyi, E. Wind Energy Handbook. John Wiley and Sons, Ltd, Chichester, UK, 2002.

[7] M. Diehl, H.G. Bock, J.P. Schlöder, R. Findeisen, Z. Nagy, and F. Allgöwer. Real-time optimization and Nonlinear Model Predictive Control of Processes governed by differential-algebraic equations. $J$. Proc. Contr., 12(4):577-585, 2002.

[8] M. Diehl, H. J. Ferreau, and N. Haverbeke. Nonlinear model predictive control, volume 384 of Lecture Notes in Control and Information Sciences, chapter Efficient Numerical Methods for Nonlinear MPC and Moving Horizon Estimation, pages 391-417. Springer, 2009.

[9] M. Diehl, R. Findeisen, S. Schwarzkopf, I. Uslu, F. Allgöwer, H.G. Bock, E.D. Gilles, and J.P. Schlöder. An Efficient Algorithm for Nonlinear Model Predictive Control of Large-Scale Systems. Part I: Description of the Method. Automatisierungstechnik, 50(12):557-567, 2002.

[10] H.J. Ferreau. An Online Active Set Strategy for Fast Solution of Parametric Quadratic Programs with Applications to Predictive Engine Control. Master's thesis, University of Heidelberg, 2006.

[11] H.J. Ferreau. Model Predictive Control Algorithms for Applications with Millisecond Timescales. $\mathrm{PhD}$ thesis, K.U.Leuven, 2011.

[12] S Gros. An Economic NMPC Formulation for Wind Turbine Control. In Conference on Decision and Control (submitted), 2013.

[13] B. Houska, H.J. Ferreau, and M. Diehl. ACADO Toolkit - An Open Source Framework for Automatic Control and Dynamic Optimization. Optimal Control Applications and Methods, 32(3):298-312, 2011.

[14] B. Houska, H.J. Ferreau, and M. Diehl. An Auto-Generated Real-Time Iteration Algorithm for Nonlinear MPC in the Microsecond Range. Automatica, 47(10):2279-2285, 2011.

[15] A. Koerber and R. King. Nonlinear Model Predictive Control for Wind Turbines. In Proc.EWEA, 2011.

[16] W.E. Leithead and B. Connor. Control of variable speed wind turbines: Design task. International Journal of Control, 73:1189-1212, 2010.

[17] I. Munteanu, A.I. Bract, N.-A. Cutululis, and E. Ceangă. Optimal Control of Wind Energy Systems. Springer, 2007.

[18] L. Y. Pao and K. E. Johnson. A Tutorial on the Dynamics and Control of Wind Turbines and Wind Farms. In Proc. Amer. Ctrl. Conf., June 2009.

[19] R. Quirynen, S. Gros, and M. Diehl. Fast auto generated ACADO integrators and application to MHE with multi-rate measurements. In Proceedings of the European Control Conference, 2013.

[20] J.B. Rawlings and B.R. Bakshi. Particle filtering and moving horizon estimation. Computers and Chemical Engineering, 30:1529-1541, 2006.

[21] D. Schlipf, A. Rettenmeier, and P.W. Cheng. Model of the Correlation between Lidar Systems and Wind Turbines for Lidar Assisted Control. In 16th International Symposium for the Advancement of BoundaryLayer Remote Sensing, Boulder, CO, 2012.

[22] D. Schlipf, D.J. Schlipf, and M. Kuehn. Nonlinear Model Predictive Control of Wind Turbines Using LIDAR. Wind Energy, 2012.

[23] M. Soliman, O.P. Malik, and D.T. Westwick. Multiple-Model MIMO Predictive Control for Variable Speed Variable Pitch WindTurbines. In Proc. American Control Conference, 2010.

[24] B. Connor W.E. Leithead. Control of variable speed wind turbines: Design task. Int. J. Control, 73(13):11891212, 2000. 